\title{
Removal of Ocular Artifacts from EEG Signals by Fast RLS Algorithm using Wavelet Transform
}

\author{
P.Ashok Babu \\ Narsimha Reddy Engineering College, \\ Hyderabad, A.P., INDIA
}

\author{
Dr.K.V.S.V.R. Prasad \\ D.M.S.S.V.H. college of Engineering \\ Machilipatnam, A.P., INDIA
}

\begin{abstract}
This paper presents an adaptive filtering method to remove ocular artifacts in the electroencephalogram (EEG) records. The major concern in analyzing EEG signal is the presence of ocular artifacts in EEG records caused due to various factors. It is essential to design specific filters to remove the artifacts in EEG records. Here, we proposed an adaptive filtering method that uses RLS (Recursive Least Square) algorithm and FRLS (Fast Recursive Least Squares) to remove ocular artifacts from EEG recordings through wavelet transform. We compared RLS \& FRLS algorithms with wavelet transforms. Elapsed time can be decreased by using the FRLS algorithm compared to other techniques and also we can compare the PSNR and MSE values.
\end{abstract}

Keywords: EEG, ocular artifacts, recursive algorithm, stationary wavelet transform, Fast recursive least squares.

\section{INTRODUCTION}

Major problem in neuroscience is the analysis of electrical recordings of the brain activity by an electroencephalogram. Eye blinks is one of the common problems faced while recording the EEG signals and the movements of eye balls produce ocular artifacts. One way to deal with this problem

is to provide subjects with a fixation point and to instruct them to make no eye movements or blinks. The effective filtering of these ocular artifacts is extremely difficult because their frequency spread $(1 \mathrm{~Hz}-50 \mathrm{~Hz})$ overlaps with that of the EEG. Various methods were proposed in recent years to correct ocular artifacts in EEG signals based on the relation between the EOG signals and the artifacts and most of these methods are non-adaptive. Croft [2] reviews a number of methods dealing with ocular artifacts in EEG focusing on relative merits of variety of EOG correction procedures. Garces Correa [3] proposed a cascade of three adaptive filters based on Least Mean Squares (LMS) algorithm to reduce the common artifacts present in EEG signals without removing significant information. Wavelet analysis provides flexible control over the resolution with which neuro-electric components and events are localized in time, space and scale. Samar [7] describes the basic concepts of wavelet analysis and other applications.

Among all the adaptive filters, recursive least squares (RLS) and fast RLS (FRLS) algorithms are well known. The latter is a computationally fast version than the former. Though RLS is not as widely used as the least mean square (LMS), it has a very significant theoretical interest as it belongs to the Kalman filters family. Many adaptive algorithms (including the LMS) are the approximations of RLS. So, there is a need to interpret and understand the different variables of RLS algorithm in different ways [11].
The convergence rate, misalignment and numerical stability of adaptive algorithms depend on the number of the input signal covariance matrix. The higher this condition number, slower the convergence rate and/or the algorithm is less stable. For ill- conditioned input signals (like speech), the LMS converges very slowly and the stability and misalignment of FRLS are more affected. So, there is a need to compute the condition number in order to monitor the behavior of adaptive filters. Unfortunately, it is difficult to estimate this condition number. In this paper we proposed an adaptive filtering technique for de-noising of these ocular artifacts using Symlet (sym3) wavelets.

\section{WAVELETS FOR ANALYZING EEG SIGNALS}

Statistical settings usually concern with discretely sampled rather than continuous functions. Wavelet transforms [1,9] have emerged as one of the superior techniques in analyzing non-stationary signals like EEG. Its capability to transform a time domain signal into frequency domain and frequency localization helps to understand the behavior of a signal better. The Discrete Wavelet Transform (DWT)[8] means choosing subsets of the scales ' $\mathrm{j}$ ' and positions ' $\mathrm{k}$ ' of the mother wavelet $\varnothing(t)$.

$$
\emptyset_{\mathrm{j}, \mathrm{k}}(\mathrm{t})=2^{\mathrm{j} / 2} \varnothing\left(2^{\mathrm{j}} \mathrm{t}-\mathrm{k}\right)
$$

Choosing scales and positions based on powers of two, which are called dyadic scales and positions ( $\mathrm{j}$ and $\mathrm{k}$ are integers). Equation (1) shows that it is possible to build a wavelet for any function by dilating a function $\varnothing(t)$ with a coefficient $2 \mathrm{j}$, and translating the resulting function on a grid whose interval is proportional to $2^{-\mathrm{j}}$. Contracted (compressed) versions of the wavelet function match the high-frequency components, while dilated (stretched) versions match the lowfrequency components. Then, by correlating the original signal with wavelet functions of different sizes, the details of the signal can be obtained at several scales. These correlations with different wavelet functions can be arranged in a hierarchical scheme called multi-resolution decomposition. The multi-resolution decomposition algorithm [5] separates the signal into "details "at different scales and a coarser representation of the signal named "approximation". The basic DWT algorithm can be modified to give a SWT [6] that no longer depends on the choice of origin. As a consequence of the sub sampling operations in the pyramidal algorithm, DWT does not preserve translation invariance. i.e., a translation of the original signal does not necessarily imply a translation of the corresponding wavelet coefficients. The SWT has been introduced in order to preserve this property. Instead of sub sampling, SWT utilizes recursively dilated filters in order to halve the bandwidth from one level to the other. This decomposition scheme is shown in figure 1. 


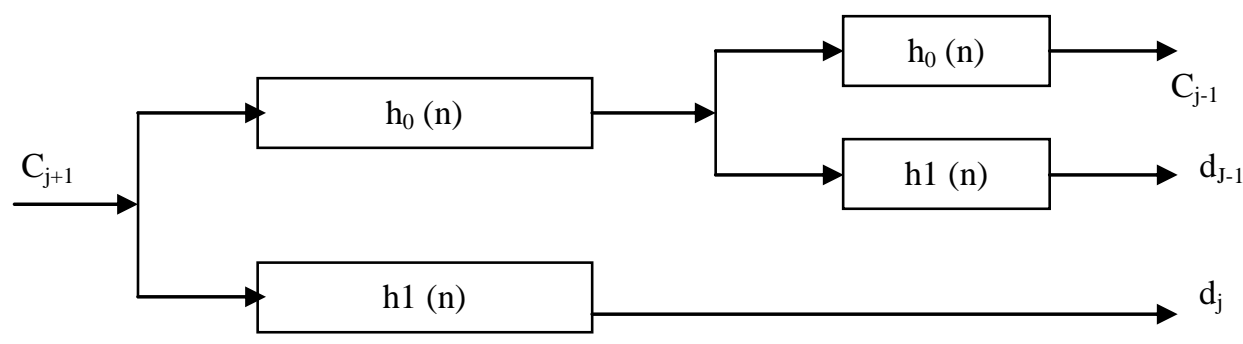

Fig. 1: Wavelet decomposition scheme

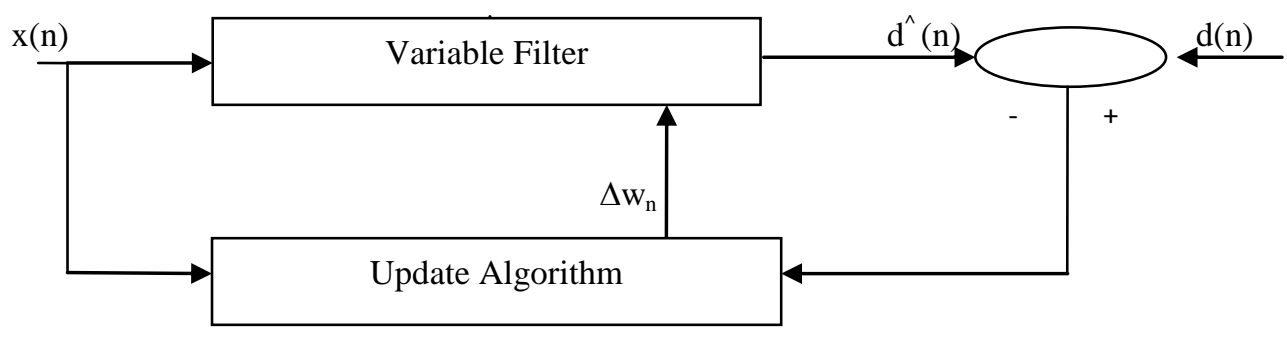

Fig. 2: Structure of an adaptive filter

\section{ADAPTIVE FILTERING}

Adaptive algorithms [10] play a very important role in many diverse applications such as communications, acoustics, speech, radar, sonar, seismology, and biomedical engineering. Adaptive-algorithms such as LMS, recursive LS or exponentially weighted LS can be used to update the coefficients of the adaptive filter. Among all the adaptive filters, Recursive Least-Squares (RLS) and Fast RLS (FRLS) algorithms are well known. FRLS is computationally less complex version of the RLS. Even though the RLS is not as widely used as the Least-Mean-Square (LMS) algorithm, it has very significant theoretical interest as it belongs to the Kalman filters family. The convergence rate, misalignment and numerical stability of adaptive algorithms depend on the number of the input signal covariance matrix [12]. Thus, there is a need in computing the condition number in order to monitor the behavior of adaptive filters.

\section{FAST RLS ALGORITHM}

The most promising classes of adaptive filtering algorithms for removal of ocular artifacts is Fast Recursive Least Squares algorithm. This algorithm exhibits computationally low complexity than RLS algorithm. The computation time of the RLS algorithm scales with $\mathrm{O}\left(\mathrm{M}^{2}\right)$, where $\mathrm{M}$ is the filter order, where as the computation time of FRLS algorithm scales with $\mathrm{O}(\mathrm{M})$. The main advantage of the RLS and FRLS algorithms is these will reduce the artifacts that are present in the EEG signals. Because the EEG signal is the low frequency signal the FRLS filter will eliminate the high frequency contents i.e. the noise that present in the EEG signal efficiently than the RLS filter. In FRLS method it will read the data and perform scaling on EEG and EOG data. For noisy and EOG data analyze the data and we find the 1 and $h$ bands. For each and every sample we applied the FRLS algorithm to do the filtering and we calculate the error in EOG signal based on reference EOG signal. We estimated the EOG signal and find out the EEG data as the difference of input signal and estimated EOG data.

\section{METHODOLOGY}

The EEG recordings are contaminated by EOG signal. The method proposed in this paper involves the following steps

(i) Apply Stationary Wavelet Transform to the contaminated EEG and reference EOG with Symlet (sym3) as the basis function and decompose the image.

(ii) Apply adaptive filter with RLS and FRLS algorithms in which the output signal is subtracted from the corrupted EEG signal to produce the artifact free EEG signal

(iii) Apply wavelet reconstruction procedure to reconstruct the EEG signal to produce the artifact free EEG signal.

\subsection{Fast RLS Algorithm with Wavelet transforms}

1).Read the data and perform scaling on EEG and EOG.

2). Evaluate sym 3 synthesis filters to populate low pass and high pass analysis.

3). For Noisy and reference EOG data, perform analysis to get ' $l$ ' and ' $h$ ' bands.

4). For each and every sample, perform fast RLS algorithm to predict and estimate the EOG sample. Then calculate error in EOG sample for updation.

5).Get EEG data as difference of original signal and estimated EOG data.

6).Calculate PSNR for original and filtered data.

7).Plot PSDs of original and filtered data 


\section{RESULTS AND DISCUSSION}

\subsection{Results for RLS Algorithm with Wavelet Transforms}

From the results we can observe that fig 3(a) is the combination of EEG, EOG, and with some noise. From the fig3(a) the black colored signal is the original EEG, the red colored signal is the EOG signal and the blue colored signal is the noisy signal. From fig 3(b) the black colored signal is the noisy EOG signal and green colored signal is the estimated EOG signal. If we subtract the estimated EOG signal from the EEG/EOG/noise EEG signal we can estimate the actual EEG signal. From fig 3(c) the black colored signal is the noisy EEG signal and green colored signal is the estimated EEG signal. From fig 3(d) the red colored signal is the noisy PSD and blue colored signal is the filtered PSD.

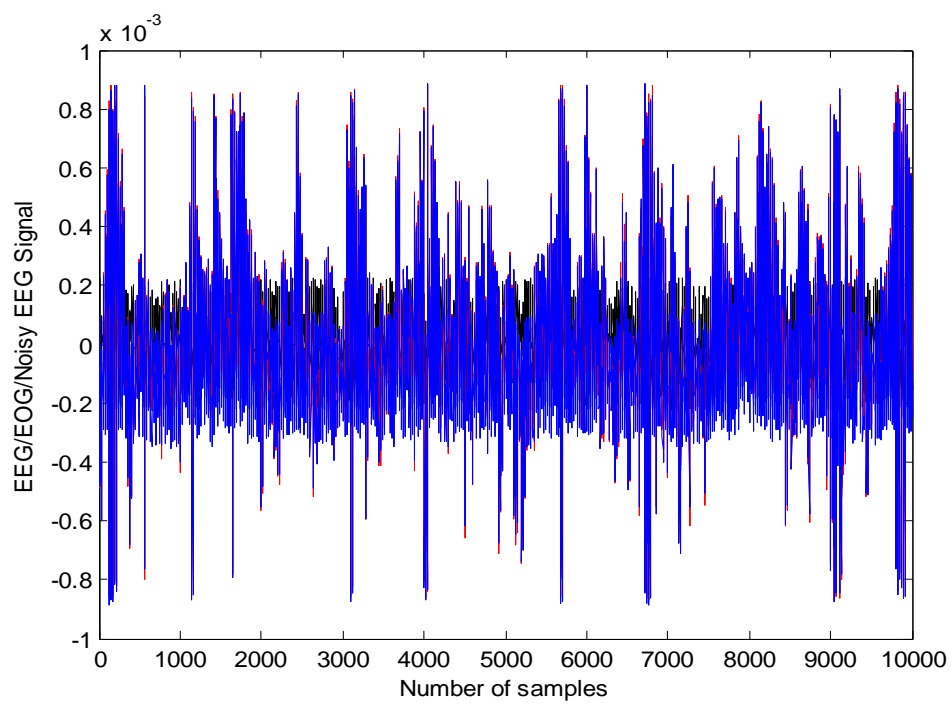

(a)

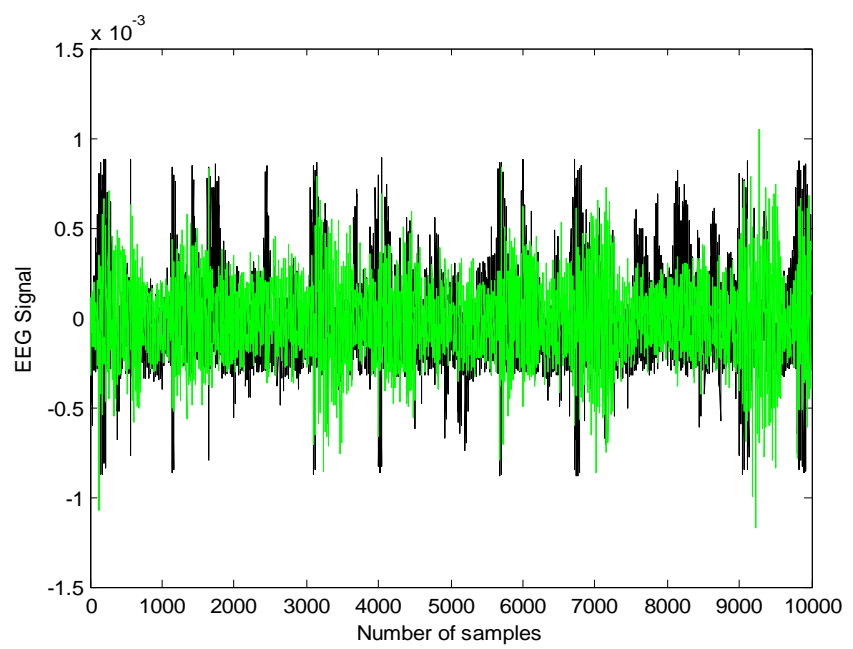

(c)

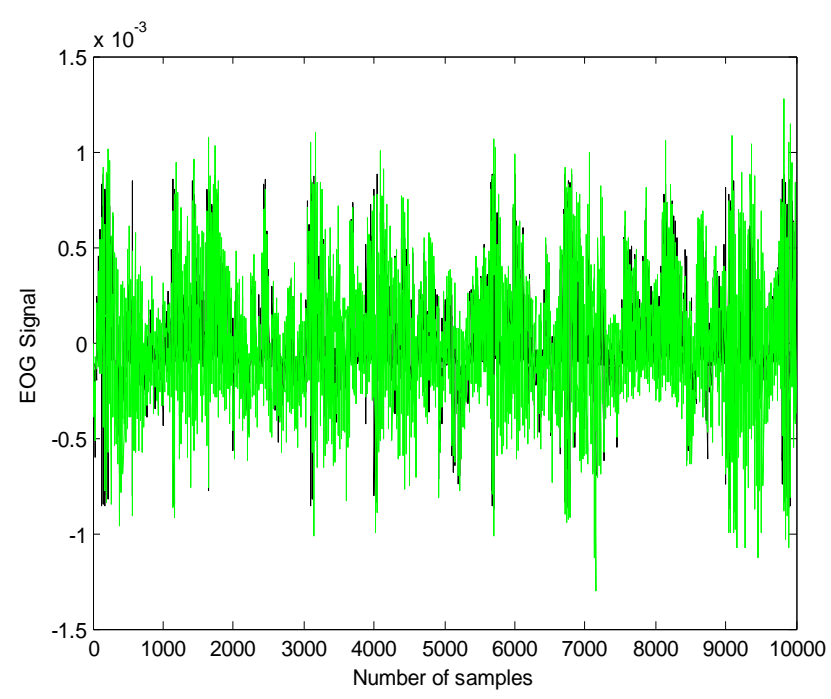

(b)

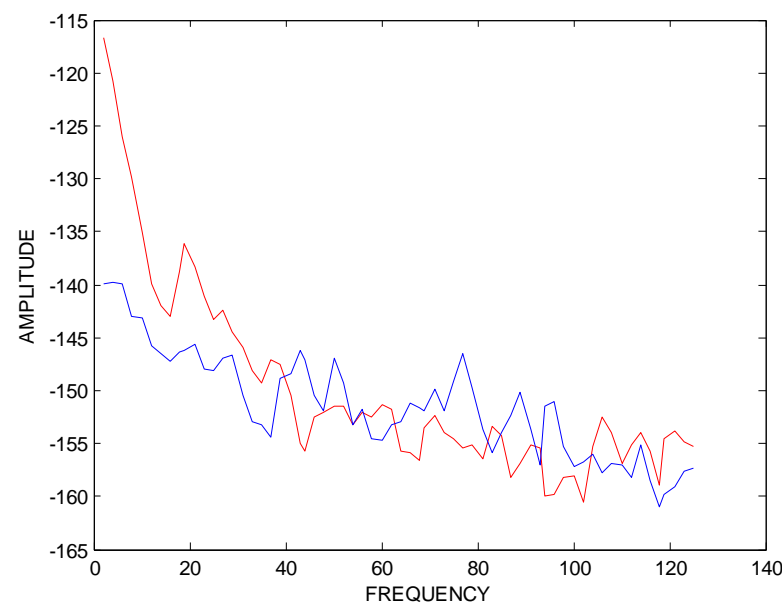

(d)

Fig.3.Results for RLS algorithm with wavelet transforms: (a) is the EEG/EOG/Noisy EEG signal, (b) is the estimated EOG signal, (c) is the estimated EEG signal, (d) is the Power spectral density curve 


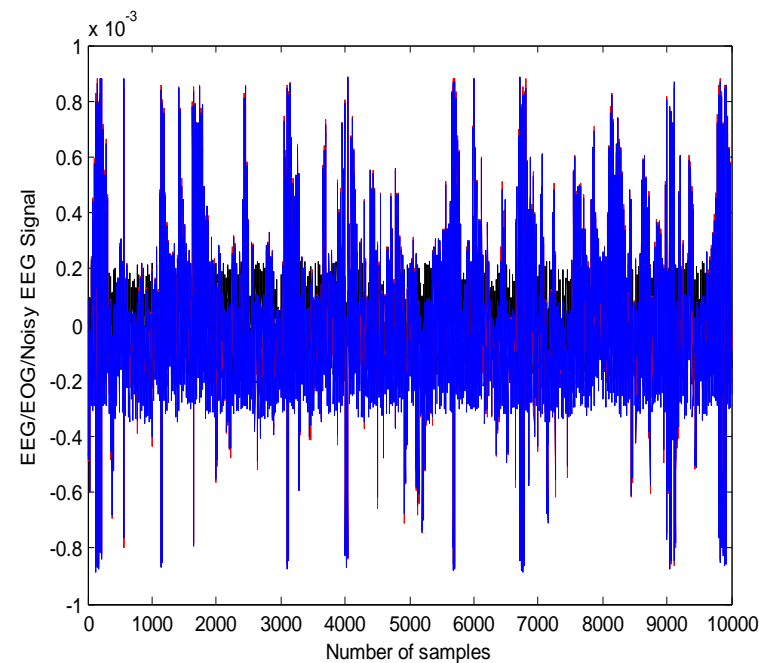

(a)

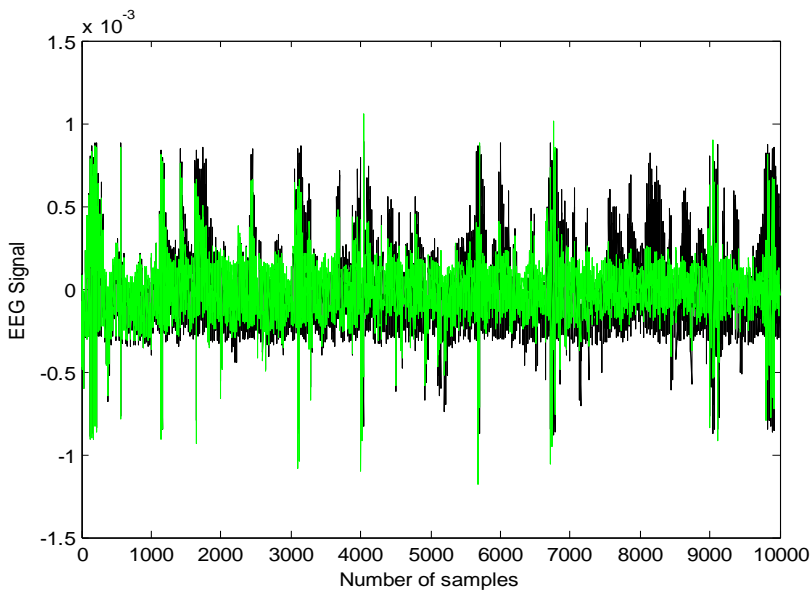

(c)

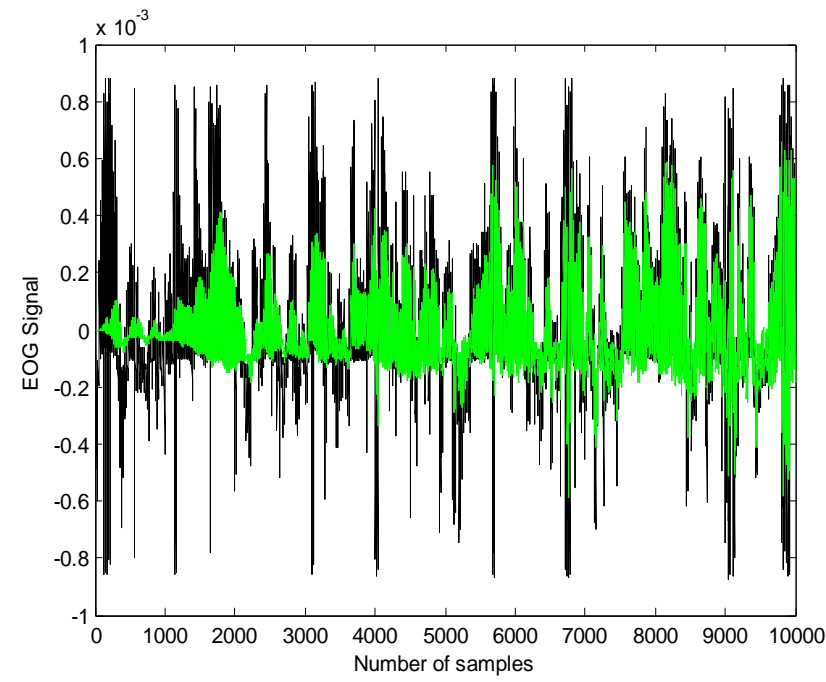

(b)

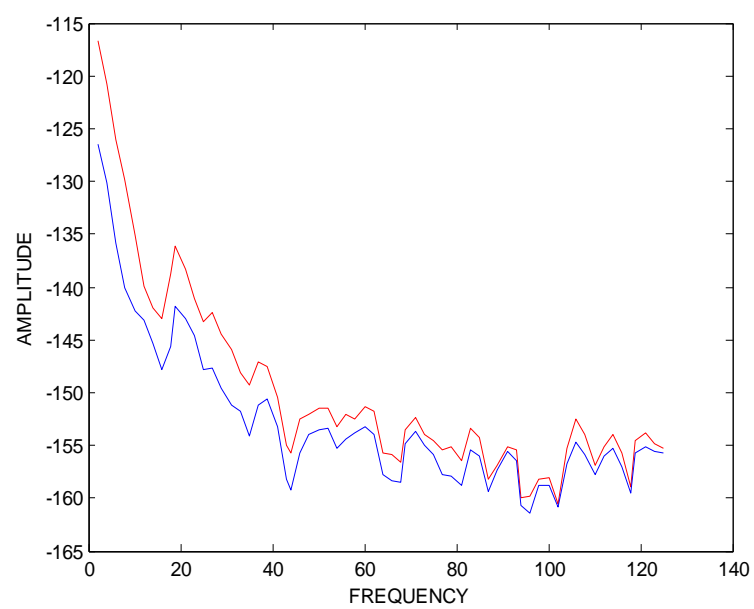

(d)

Fig.4.Results for FRLS algorithm with wavelet transforms: (a) is the EEG/EOG/Noisy EEG signal, (b) is the estimated EOG signal, (c) is the estimated EEG signal,(d) is the Power spectral density curve

\subsection{Results for Fast RLS Algorithm with Wavelet Transforms}

From the results we can observe that fig $4(a)$ is the combination of EEG, EOG, and with some noise. From the fig4(a) the black colored signal is the original EEG, the red colored signal is the EOG signal and the blue colored signal is the noisy signal. From fig 4(b) the black colored signal is the noisy EOG signal and green colored signal is the estimated EOG signal. If we subtract the estimated EOG signal from the EEG/EOG/noise EEG signal we can estimate the actual EEG signal. From fig 4(c) the black colored signal is the noisy EEG signal and green colored signal is the estimated EEG signal. From fig 4(d) the red colored signal is the noisy PSD and blue colored signal is the filtered PSD

Table1: Comparison of MSE, PSNR and Elapsed Times

\begin{tabular}{|c|c|c|c|c|c|}
\hline Algorithm & $\begin{array}{c}\text { Noisy - } \\
\text { MSE }\end{array}$ & $\begin{array}{c}\text { Filtered- } \\
\text { MSE }\end{array}$ & $\begin{array}{c}\text { Noisy- } \\
\text { PSNR }\end{array}$ & $\begin{array}{c}\text { Filtered- } \\
\text { PSNR }\end{array}$ & Elapsed Time \\
\hline RLS Algorithm & $\begin{array}{c}6.5806 \mathrm{e}- \\
008\end{array}$ & $\begin{array}{c}4.6227 \mathrm{e}- \\
008\end{array}$ & 0.8704 & 3.9379 & $12.152955 \mathrm{sec}$ \\
\hline $\begin{array}{c}\text { RLS Algorithm } \\
\text { with wavelets }\end{array}$ & $\begin{array}{c}6.5806 \mathrm{e}- \\
008\end{array}$ & $\begin{array}{c}3.3760 \mathrm{e}- \\
008\end{array}$ & 0.8704 & 6.6677 & $10.170116 \mathrm{sec}$ \\
\hline $\begin{array}{c}\text { FRLS } \\
\text { Algorithm with } \\
\text { wavelets }\end{array}$ & $\begin{array}{c}6.5806 \mathrm{e}- \\
008\end{array}$ & $\begin{array}{c}2.5635 \mathrm{e}- \\
008\end{array}$ & 0.8704 & 9.0591 & $1.695036 \mathrm{sec}$ \\
\hline
\end{tabular}


From the table we compared PSNR and Elapsed times for RLS and Fast RLS algorithms. We consider the noisy PSNR as 0.8704 for all the signals and the filtered PSNR values for RLS and FRLS algorithms are 6.6677 and 9.0591.The Elapsed time decreases in case of FRLS compared to RLS algorithm and the values are $10.170116 \mathrm{sec}$ for RLS and $1.695036 \mathrm{sec}$ for FRLS algorithms. From the table we can conclude that FRLS algorithm is more efficient compared to RLS algorithm.

\section{CONCLUSION}

Our proposed method using adaptive filter with Fast RLS algorithm through wavelet transform reduces the artifacts in EEG compared to RLS algorithm with wavelet transforms. It is an efficient technique to improve the quality of EEG signal and also increases the PSNR value and decreases the elapsed time compared to RLS algorithm. We conclude that adaptive cancellation with help of wavelet decomposition can be considered to be a preprocessed work and is an efficient processing technique for improving the quality of EEG signals in biomedical analysis.

\section{REFERENCES}

[1]. Akay, M. 1998."Time Frequency andWavelets in Biomedical Signal Processing", IEEE Press series in biomedical Engineering

[2]. Croft, R.J. and R.J. Barry, 2000. "Removal of ocular artifact from the EEG: a review", Clinical Neurophysiology, 30(1): 5 - 19.

[3]. Garces, A., Correa, E. Laciar, H.D. Patino and M.E. Valentinuzzi, 2007. "Artifact removal from EEG Signals using adaptive filters in cascade", 16 Argentine Bioengineering the Congress and the 5th Conference on Clinical Engineering, 90:1-10.

[4]. Goldberger, A.L., L.A.N. Amaral, L. Glass, J.M. Hausdorff, P.C.h. Ivanov, R.G. Mark, J.E. Mietus, G.B. Moody, C.K..Peng, H.E. Stanley, 2000. Physiobank, PhysioToolkit PhysioNet: Components of a New Research Resource for Complex Physiologic Signals. Circulation 101(23):e215-e220 Circulation Electronic, Webpage: http://circ.ahajournals.org/cgi/content/full/ 101/23/e215;

[5]. Mallat, S.G. 1989. “A Theory for Multiresolution signal decomposition: The Wavelet representation".IEEE Trans. On Pattern Analysis and Machine Intell. 2(7) 674 $-693$

[6]. Nason, G.P. and B.W. Silverman, 1995. "The Stationary Wavelet Transform and some Statistical Applications", Tech. Rep. BS8 1Tw, University of Bristol.
[7]. Samar, V. J., 1999. Ajit Bopardikar, Raghuveer Rao, and Kenneth Swartz, "Wavelet analysis of neuroelectric waveforms: A conceptual tutorial", Brain and Language, 66: $7-60,1999$.

[8]. Sidney, C. 1998. Burrus, Ramesh A Gopinath and Haitao Guo, "Introduction to wavelets and wavelet transforms", Prentice-Hall international Inc.

[9]. Tompkins, W.J. 1993. "Biomedical digital signal processing”, New Jersey, Prentice Hall.

[10]. Widrow, B. and S.D. Stearns, 1985. "Adaptive Signal Processing”, New Jersey, Prentice Hall.

[11]. P.senthil Kumar, R. Armuganathan,K.Sivakumar, C.Vimal,2009. "An Adaptive method to remove ocular Artifacts from EEG signals using Wavelet Transforms", Journal of Applied Science and Research.

[12]. Jacob Benesty, Tomas G" ansler, "New Insights into the RLS Algorithm", EURASIP Journal on Applied Signal Processing 2004:3, 331-339

\section{AUTHOR BIOGRAPHIES}

${ }^{1}$ Mr.P.Ashok Babu obtained B.E Degree in 2001 from Andhra University, M.E (Communication Engineering) in 2005 from Osmania University. He pursuing the Ph.D. from JNTUH university, Hyderabad in Digital Image Processing . He published two papers in International journals and one paper in International Conference. He is member of IACSIT and IAENG. Presently he is working as Assoc. Professor and Head, Department of ECE, Narasimha Reddy Engineering College, Dhullapally, Hyderabad, Andhra Pradesh (state) India.

${ }^{2}$ Dr.K.V.S.V.R Prasad obtained BSc. Degree in1963from AndhraUniversity, B.E(Telecommunication Engineering)in 1967andM.E(ECE)Microwave Engineering specialization in 1977 from Osmania University. He received the Ph.D. in 1985 from IIT Kharagpur in strip and micro strip transmission lines. He published six papers in IEEE Transactions in MTT, Antenna and Propagation and EMI/EMC and three papers in National Conferences. He is fellow of IETE (life member). He worked in various capacities in the Department of ECE, Osmania University, Hyderabad. Presently he is working as professor and head, Department of ECE, D.M.S.S.V.H. College of engineering, Machilipatnam, Andhra Pradesh (state), India. 